\title{
A Survey on High Utility Rare Itemset Mining
}

\author{
Zhang Wenbo ${ }^{1}$, Kanika Middha ${ }^{2}$ and Jeetesh Kumar Jain ${ }^{3}$ \\ ${ }^{1}$ Department of Information Science and Engineering, Shenyang Ligong University, \\ Shenyang, Liaoning, China \\ ${ }^{2,3}$ Computer Science and Engineering, SRCEM, Benmore, Gwalior, M. P., India \\ ${ }^{1}$ wenbo_sy@163.com, ${ }^{2} J a i n j e e t e s h 9 @ g m a i l . c o m,{ }^{3}$ kanikabeit@gmail.com
}

\begin{abstract}
In this paper, a survey about basic data mining and its related fields like association rule mining has been undertaken. In our daily life, we come across a lot many things that have profit (external utility) value greater than the other itemsets in the database. Thus, a new concept that needs new work done is utility mining. Rare itemsets have been explained that are used for the mining of the items having utility value less than the threshold but are of great use. A brief literature survey is given to show the work done in this field to date.
\end{abstract}

Keywords: Data mining, Association rule mining, Utility mining, Rare itemsets

\section{Introduction}

Many business enterprises accumulate large quantities of data from day-to-day operations. For example, a huge amount of customer purchase data are collected daily at the checkout counters grocery stores. Retailers are interested in analyzing the data to learn about the purchasing behavior of the customers. Such valuable information can be used to support a variety of business-related applications such as marketing promotions, inventory management, and customer relations management [1].

Data mining techniques can be used to support a wide range of business intelligence applications such as customer profiling, targeted marketing, workflow management, store layout, and fraud detection. It can also help retailers answer important business questions such as the most important customers or most profitable transactions, etc.

Over the years Data Mining is used to understand consumer buying behavior using various techniques. The researcher selects Market Basket Analysis for his data analysis because MarketBasket analysis is a tool of knowledge discovery about the co-occurrence of nominal or categorical items. Market Basket Transaction or Market Basket Analysis is a data mining technique to derive associations between data sets. The discovery of interesting association relationships among huge amounts of customer transaction records can help in many business decision-making processes such as catalog design, cross-marketing, and loss-leader analysis[2].

Data mining refers to extracting or mining knowledge from large amounts of data. Thus, data mining should have been more appropriately named "knowledge mining from data". The task of finding frequent patterns in data mining in large databases is very important to use full in many applications over the past few years. The primary goal is to discover hidden patterns, unexpected trends in the data. Data mining is concerned with the analysis of large volumes of

\section{Article history:}

Received (January 15, 2015), Review Result (February 18, 2015), Accepted (April 26, 2015) 
data to automatically discover interesting regularities or relationships which in turn leads to a better understanding of the underlying processes. Data mining activities use a combination of techniques from database artificial intelligence, statistics, technology machine learning. This includes bioinformatics, genetics, medicine, clinical research, education, retail, and marketing research [3].

Data Mining involves an algorithmic process, which takes preprocessed input data and extracts patterns. Various techniques exist such as association rule mining, classification, clustering, etc. An important and widely used data mining technique is the discovery of association rules. Association rule mining aims at discovering frequent itemsets from market basket data and generating association rules. Most association rule mining algorithms implicitly consider the utilities of the itemsets to be equal [Yao H. et al (2004)] [4]. A utility is a value attached to an item depending on its evaluation e.g. if coke has support 20 and profit of $2 \%$, cookies may have support 10 but with a profit of $20 \%$.

\subsection{Association rule mining}

Several techniques are satisfying these objectives of data mining. Mining Associations are one of the techniques involved in the process mentioned above and among the data mining problems, it might be the most studied ones. Discovering association rules is at the heart of data mining. Mining for association rules between items in a large database of sales transactions has been recognized as an important area of database research. These rules can be effectively used to uncover unknown relationships, producing results that can provide a basis for forecasting and decision making. The original problem addressed by association rule mining was to find a correlation among sales of different products from the analysis of a large set of data[5]. Association rule mining (ARM) is the process of generating rules based on the correlation between the set of items that the customers purchase. Of late, data mining researchers have improved upon the quality of association rule mining for business development by incorporating factors like value (utility), the number of items sold (weight), and profit. The rules mined without considering utility values (profit margin) will lead to a probable loss of profitable rules. Mining Association rules are one of the examination issues in information (data) mining. a set of transactions where every exchange is a set of items, an association principle is a statement of the structure $\mathrm{X} \mathrm{Y}$, where $\mathrm{X}, \mathrm{Y}$ (sets of items). The issue of mining association rule was initially presented in and later widened in, for the instance of databases comprising of categorical qualities alone. As information keeps on growing and its complexity increments, date information structures and calculations are being produced to match this improvement (development). Association Rule Mining procedure can be partitioned into two stages. The first step includes discovering all large itemsets (or frequent itemsets) in DB (database). When the frequent itemsets are discovered, association rules are created. Association standard mining is broadly utilized as a part of market-basket investigation[6].

In general, the association rule is an expression of the form $X=>Y$, where $X$ is antecedent and $\mathrm{Y}$ is consequent. The Association rule shows how many times $\mathrm{Y}$ has occurred if $\mathrm{X}$ has already occurred depending on the support and confidence value. Support: It is the probability of item or itemsets in the given transactional database: $\operatorname{support}(X)=n(X) / n$ where $n$ is the total number of transactions in the database and $n(X)$ is the number of transactions that contain the item set $X$.Therefore, support $(X=>Y)=\operatorname{support}(X U Y)$. Confidence: It is conditional probability, for an association rule $X=>Y$ and defined as

confidence $(X=>Y)=\operatorname{support}(X U Y) / \operatorname{support}(X)$. Frequent itemset: Let $A$ be a set of items, $\mathrm{T}$ be the transaction database and $\sigma$ be the user-specified minimum support. An itemset $\mathrm{X}$ in 
A (i.e., $\mathrm{X}$ is a subset of $\mathrm{A}$ ) is said to be a frequent itemset in $\mathrm{T}$ concerning $\sigma$ if $\operatorname{support}(\mathrm{X}) \mathrm{T}>=$ $\sigma$. Mining association rules can be broken down into the following two sub-problems:

1. Generating all itemsets that have supported greater than, or equal to, the user-specified minimum support. That is, generating all large itemsets. 2. Generating all the rules that have minimum confidence. We can generate the association rule with more than one number of consequent items is generated by the following method: 1 . Find the rule in which the number of consequents $=1$.

2. For the given rules $p(x->y)$ and $p(x->z)$, the rule $p(x->y z)$ is generated by the intersection of both the association rules and get a new rule $p(x->y z)=p(x y z) / p(x)$.

\subsection{Rare itemset mining}

In this section, we present an example of rare and non-present item-set mining. The input data is composed of a database of transactions, and each transaction is identified by an id and composed of a set of items. In the real world, a transaction may be seen as the basket bought by a customer during a determined period (day, week, month, etc.). Each basket is composed of a set of items that are purchased together. In Table 1 we represent an abstract database, denoted by $\mathrm{D}$, where the alphabet letters are considered as items. Given the database of transactions such as presented in [Table 1], our goal is to find two categories of sets of items, also called item sets. The first category is composed of itemsets that are present in at most two transactions, and the second category is composed of itemsets that do not occur in any transaction and composed of a maximum number of items equal to the cardinality of the largest transaction. The number of times an item-set occurs in the database is called the itemset support. In our case, the maximum support is equal to 3[7].

Table 1. Transaction database

\begin{tabular}{|c|c|}
\hline ID & Traqnsaction \\
\hline $\operatorname{Tr} 1$ & $\{\mathrm{a}, \mathrm{b}, \mathrm{c}, \mathrm{d}\}$ \\
\hline $\operatorname{Tr} 2$ & $\{\mathrm{~b}, \mathrm{~d}\}$ \\
\hline $\operatorname{Tr} 3$ & $\{\mathrm{a}, \mathrm{b}, \mathrm{c}, \mathrm{e}\}$ \\
\hline $\operatorname{Tr} 4$ & $\{\mathrm{c}, \mathrm{d}, \mathrm{e}\}$ \\
\hline $\operatorname{Tr} 5$ & $\{\mathrm{a}, \mathrm{b}, \mathrm{c}\}$ \\
\hline
\end{tabular}

The set of all itemsets that can be generated from the transaction database is presented in Figure 1 by a diagram of the subset lattice for five items with the associated frequencies in the database. In the lattice, each level is composed of itemsets having the same length. The top element in the lattice is the empty set.

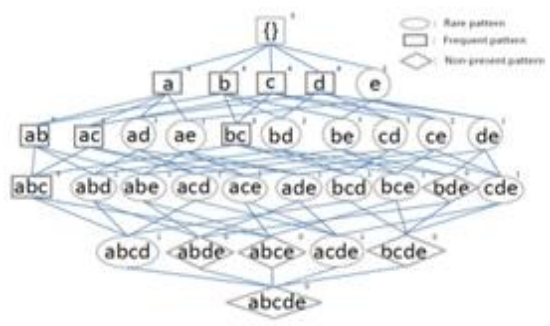

Figure 1. 
Figure 1: Lattice represents a hierarchically ordered space of itemsets and their frequencies. Frequent itemsets are square-shaped, rare itemsets are oval-shaped and non-present itemsets are diamond-shaped.

Each lower level $\mathrm{k}$ contains all of the item-sets of length $\mathrm{k}$, also denoted $\mathrm{k}$-item-sets and the last level contains an item-set composed of all items (i.e. a, b, c, d, e). Lines between nodes represent a subset relationship between item-sets. For each item-set we compute its support. For example, the item-set composed of the items $b$ and denoted by have 2 as support, and we denote it by . In fact, the item-set bd is present in the transactions $t 1, t 2$. The set of rare item-sets we are looking for are those in the lattice with support greater than 0 and less than 3. The rest of item-sets are either frequent (having support greater or equal to 3 ) or non-present (support=0). In Figure 1 rare item-sets are drawn with ovals, frequent item-sets are drawn with rectangles where non-present item-sets are drawn with diamonds. Thus, after counting the frequencies of each item-set, we obtain the following set of rare item-sets $\langle e, 2\rangle$, $\langle\mathrm{ad}, 1\rangle,\langle\mathrm{ae}, 1\rangle,\langle\mathrm{bd}, 2\rangle,\langle\mathrm{be}, 1\rangle,\langle\mathrm{cd}, 2\rangle,\langle\mathrm{de}, 1\rangle,\langle\mathrm{abd}, 1\rangle,\langle\mathrm{abe}, 1\rangle,\langle\mathrm{acd}, 1\rangle,\langle\mathrm{ace}, 1\rangle$, $\langle$ ade, 2$\rangle,\langle$ bcd, 1$\rangle,\langle$ bce, 1$\rangle,\langle$ cde, 1$\rangle,\langle$ abcd, 1$\rangle,\langle$ acde, 1$\rangle$.

\subsection{Utility mining}

The conventional Association standard mining methodologies consider the utility of the items by their presence in the exchange (transaction) set. The recurrence of an itemset is not adequate to mirror (reflect) the real utility of an itemset. For instance, the business administrator may not be occupied with continuous itemsets that don't create significant benefits. Recently, one of the most difficult information mining undertakings is the mining of high utility itemsets productively[8]. Distinguishing proof of the itemsets with HU (high utilities) is called UM (Utility Mining). The utility can be measured as far as expense, benefit, or different articulations of client inclinations.

Information mining is the procedure of uncovering nontrivial, previously unknown, and conceivably helpful data from huge (large) databases. an essential role in several data mining tasks, such as frequent pattern mining, weighted frequent pattern mining, and high utility pattern mining play for Discovering useful patterns hidden in a database. Among them, continuous pattern mining is essential. Mining high utility itemsets from databases allude (refers) to discovering the itemsets with high benefits. Here, the significance of itemset utility is the interestingness, significance, or profitability of an item to clients. Utility of item in exchange (transaction)DB comprises of two perspectives:

1) The significance of particular items, which is known as external utility, and

2) the significance of items in communications, which is known as internal utility.

Utility mining of an item set is characterized as the result of its external utility and its internal utility. An itemset is known as a high utility mining item set its utility is no less than a client determined least utility edge; otherwise, it is known as a low-utility itemset. Mining high utility itemsets from databases is a critical undertaking has an extensive variety of utilizations, for example, site snap stream examination business advancement in chain hypermarkets, cross-advertising in retail locations, online e-trade administration, and portable business environment arranging, and even discovering an imperative pattern in biomedical applications [9]. 
Table 2. Transaction database

\begin{tabular}{|c|c|c|c|}
\hline Transaction Id & X & Y & Z \\
\hline $\operatorname{Tr} 1$ & 1 & 1 & 1 \\
\hline $\operatorname{Tr} 2$ & 2 & 1 & 0 \\
\hline $\operatorname{Tr} 3$ & 3 & 0 & 2 \\
\hline $\operatorname{Tr} 4$ & 1 & 2 & 1 \\
\hline $\operatorname{Tr} 5$ & 0 & 1 & 0 \\
\hline $\operatorname{Tr} 6$ & 5 & 3 & 4 \\
\hline $\operatorname{Tr} 7$ & 2 & 2 & 0 \\
\hline $\operatorname{Tr} 8$ & 3 & 1 & 1 \\
\hline $\operatorname{Tr} 9$ & 4 & 1 & 1 \\
\hline $\operatorname{Tr} 10$ & 2 & 0 & 2 \\
\hline
\end{tabular}

Table 3. Unit profit associated with items

\begin{tabular}{|c|c|}
\hline Item Name & Profit \\
\hline $\mathrm{X}$ & 3 \\
\hline $\mathrm{Y}$ & 10 \\
\hline $\mathrm{Z}$ & 8 \\
\hline
\end{tabular}

\section{Related work}

In this section, the related work that has been done till now on rare itemset mining is shown. Various algorithms that have been implemented are explained:

R. Agrawal et al in [10] proposed the Apriori algorithm, which is used to obtain frequent itemsets from the database. The itemsets which appear frequently in the transactions are called frequent itemsets. MINIT (MINimal Infrequent Itemsets), which is the first algorithm designed specifically for mining minimal infrequent itemsets (MIIs)[11]. A minimal infrequent item-set is an infrequent item-set that does not have a subset of items that forms an infrequent item-set. MINIT is both minimal and non-minimal (unweighted) infrequent itemset mining from unweighted data. It is based on the SUDA2 algorithm. Also showed that the minimal infrequent itemset problem is an NP-complete problem.

The author in [12] has purposed a way to find out weights of items and weights of transactions without pre-assigned weights in the database. HITS model is used to derive the weights of transactions from a database with only binary attributes which is based on Link Ranking Analysis. Based on these weights, w-support is defined to give the significance of itemsets which differs from the traditional support in considering the quality of transactions. An Apriori-like algorithm is proposed to extract association rules whose w-support and wconfidence are above some given thresholds.

Mehdi Addaet. al. described the ARANIM algorithm for Apriori Rare and Non-Present Item-set Mining to mine rare and nonpresent itemsets in [7]. Nonpresent items are used to detect what is missing in the defective process. The proposed approach is Apriorilike and the mining idea behind it is that if the item-set lattice representing the itemset space in classical Apriori approaches is traversed in a bottom-up manner, equivalent properties to the Apriori exploration of frequent item-sets is provided to discover rare item-sets[13]. Also, the author proposed an approach based on rare patterns to detect suspicious uses and behaviors in the context of a Web application. 
[14] is used to find the infrequent patterns and non-present patterns but it does not consider any pruning strategy. It is better to implement any pruning strategy to improve the complexity of the proposed method. Paper [15] proposed Talky-G and Walky-G algorithms. It uses a depth-first strategy for traversal.IFP_min algorithm described in [16] uses the concept of the residual tree to reduce computation time. The IFP_min algorithm recursively mines the minimally infrequent itemsets (MIIs) by dividing the IFP-tree into two sub-trees: projected tree and residual tree[16]. Pattern-growth-based algorithms are computationally faster on dense datasets.

Apriori-rare is a modification of the Apriori algorithm used to mine frequent itemsets. To retrieve all rare itemsets from minimal rare itemset (mRIs), a prototype algorithm called -A Rare Itemset Miner Algorithm (Arima)\| was proposed in [17]. Arimagenerates the set of all rare itemsets, splits into two sets: the set of rare itemsets having zero support and the set of rare itemsets with non-zero support. If an item set is rare then any extension of that item set will result from a rare item set [17].

Paper [18] presented WSFI (Weighted Support Frequent Itemsets) algorithm where the user can specify the weight for each item. A WSFP-Tree store compressed important information about frequent patterns which is an extended FP-tree. It mines the frequent itemsets in only one scan from the data stream.

In [19] proposed FPGrowth- like algorithms i.e. IWI and MIWI for discovering infrequent itemsets by using weights for differentiating between relevant items and not within each transaction. The IWI-support measure is defined as a weighted frequency of occurrence of an item set in the analyzed data. Occurrence weights are derived from the weights associated with items in each transaction by applying a given cost function. They mainly focus on IWIsupport-min measure and IWIsupport-max measure which are described as : (i) The IWIsupport-min measure, which relies on a minimum cost function, i.e., the occurrence of an itemset in a given transaction is weighted by the weight of its least interesting item, (ii) The IWIsupport-max measure, which relies on a maximum cost function, i.e., the occurrence of an itemset in a given transaction is weighted by the weight of the most interesting item. As per the analysis of [20], MIWI is the most effective algorithm, which computes in very less computing time, improves the efficiency of performance when the database is large, computes the weighted transaction among the existing algorithms[21].

Laszlo Szathmary, Amedeo Napoli, Petko Valtchev [22], based on an examination of the relevant substructures of the mining space, our approach splits the rare itemset mining task into two steps, i.e., frequent itemset part traversal and rare itemset listing. We propose two algorithms for step one, a naive and an optimized one, respectively, and another algorithm for step two[22].

G.C.Lan et al proposed a new kind of pattern, named Rare Utility Itemsets in [22], which considers not only individual profits and quantities but also common existing periods and branches of items in a multi-database environment. A new mining approach called TP-RUIMD (Two-Phase Algorithm for Mining Rare Utility Itemsets in Multiple Databases) was proposed to efficiently discover rare utility itemsets. The TP-RUI-MD algorithm is designed to find the rare-utility itemsets in a multi-database environment. The TP-RUI-MD algorithm uses the level-wise technique for discovering the rare-utility itemsets in a multi-database environment. The TP-RUI-MD algorithm cannot discover all rare-utility itemsets in a multidatabase environment, but the TP-RUI-MD algorithm can still discover these rare-utility undiscovered by existing algorithms in a single database environment[22].In paper [17], L. Szathmary et al presented a novel method for computing all rare itemsets by splitting the rare itemset mining task into two steps. The first step is the identification of the minimal rare 
itemsets. These itemsets jointly act as a minimal generation seed for the entire rare itemset family. In the second step, the minimal rare itemsets are processed to restore all rare itemsets. Two algorithms were proposed for the first step: (i) a naive one that relies on an Apriori-style enumeration, Apriori-Rare, and (ii) an optimized method that limits the exploration to frequent generators only. The second task is solved by a straightforward procedure. Apriorirare is a modification of the Apriori algorithm used to mine frequent itemsets. Apriori-rare generates a set of all minimal rare generators, also called MRM, that correspond to the itemsets usually pruned by the Apriori algorithm when seeking frequent itemsets. To retrieve all rare itemsets from minimal rare itemset (mRIs), a prototype algorithm called "A Rare Itemset Miner Algorithm(Arima)" was proposed. Arima generates the set of all rare itemsets, splits it into two sets: the set of rare itemsets having zero support and the set of rare itemsets with non-zero support. If an itemset is rare then any extension of that itemset will result from a rare itemset[17]

M. Adda et al proposed a framework in [24], to represent different categories of interesting patterns and then instantiate it to the specific case of rare patterns. A generic framework was presented to mine patterns based on the Apriori approach. The generalized Apriori framework was instantiated to mine rare itemsets. The resulting approach is Apriorilike and the mine idea behind it is that if the itemset lattice representing the itemset space in classical Apriori approaches is traversed on a bottom-up manner, equivalent properties to the Apriori exploration of frequent itemsets are provided to mine rare itemsets. The Apriori algorithm called AfRIM for Apriori Rare itemset to mine rare itemsets was proposed which performs a level-wise descent. The backward traversal method is endowed with a property that leads to pruning out potentially non-rare itemsets in the mining process. This includes an antimonotone property and a level-wise exploration of the itemset space

RP-Tree: Rare Pattern Tree Mining All current algorithms for rare association rule mining use an Apriori level-wise approach which has computationally expensive candidate generation and pruning steps. We propose RP-Tree, a method for mining a subset of rare association rules using a tree structure, and an information gain component that helps to identify the more interesting association rules. Empirical evaluation using a range of realworld datasets shows that RP-Tree itemset and rule generation is more time-efficient than modified versions of FP-Growth and ARIMA, and discovers 92-100\% of all the interesting rare association rules are itemset mining is very important as rare itemsets may bring adequate profits to the business. In [26], Jyothi et al proposed High Utility Rare Itemset Mining [HURI] to find high utility rare-itemsets based on minimum threshold values and user preferences. The utility of items is decided by considering factors such as profit, sale, temporal aspects, etc. of items. [26]

High Utility itemsets may contain frequent as well as rare itemsets. Classical utility mining only considers items and their utilities as discrete values. In real-world applications, such utilities can be described by fuzzy sets. Thus itemset utility mining with fuzzy modeling allows item utility values to be fuzzy and dynamic over time. In this paper, an algorithm, FHURI (Fuzzy High Utility Rare Itemset Mining) is presented to efficiently and effectively mine very-high (and high) utility rare itemsets from databases, by fuzzification of utility values. FHURI can effectively extract fuzzy high utility rare itemsets by integrating fuzzy logic with high utility rare itemset mining. FHURI algorithm may have practical meaning to real-world marketing strategies. The results are shown using synthetic datasets[27]. 


\section{Conclusion}

In DM (Data Mining), ARM (Association Rule Mining) is a standout amongst the most vital assignments. The mining can be connected to a database having any size. A substantial (LARGE) number of effective calculations are accessible for association rule mining, which considers mining of continuous Itemsets. In case, a developing subject in Data Mining is Utility Mining, which joins utility considerations during set mining. UM (Utility Mining), covers all parts of economic utility in information mining and helps in the location of uncommon things set having high utility. High Utility itemset mining is exceptionally valuable in several real-life applications.

\section{References}

[1] P.N. Tan, M. Steinbach, and V. Kumar, "Introduction to Data mining”, (2009)

[2] A. Raorane and R.V. Kulkarni, "Data Mining Techniques: A Source For Consumer Behavior Analysis", International Journal of Database Management Systems, vol.3, no.3, Aug., pp.45-56, (2011)

[3] S. Bhattacharya and D. Dubey, "High Utility Itemset Mining", International Journal of Emerging Technology and Advanced Engineering, vol.2, no.8, August (2012)

[4] H. Yao and H.J. Hamilton, "Mining itemset utilities from transaction databases," Data and Knowledge Engineering, vol.59, pp.603-626, (2006)

[5] F.H. AL-Zawaidah and Y.H. Jbara, "An Improved Algorithm for Mining Association Rules in Large Databases", World of Computer Science and Information Technology Journal (WCSIT), vol.1, no.7, pp.311316, (2011)

[6] E. Duneja and A.K. Sachan, "A Survey on Frequent Itemset Mining with Association Rules”, International Journal of Computer Applications, vol.46,no.23, May (2012)

[7] M. Adda, L. Wu, S. White, and Y. Feng, "Pattern Detection with Rare Item-Set Mining", International Journal on Soft Computing, Artificial Intelligence, and Applications (IJSCAI), vol.1, no.1, August (2012)

[8] M. Liu and J. Qu, "Mining High Utility Itemsets Without Candidate Generation", CIKM '12 Proceedings of the 21st ACM International Conference on Information and Knowledge Management, pp.55-64, (2012)

[9] W. Song, Y. Liu, and J. Li, "Mining high utility itemsets by dynamically pruning the tree structure", Applied Intelligence, January, vol.40, no.1, pp 29-43, (2014)

[10] R. Agrawal, T. Imielinski, and Swami, "Mining Association Rules between Sets of Items in Large Databases", Proceedings of the ACM SIGMOD International Conference on Management of Data (SIGMOD '93), pp. 207-216

[11] S. Khare, and Prof. V. Jain, "A Review on Infrequent Weighted Itemset Mining Using Frequent Pattern Growth", (IJCSIT) International Journal of Computer Science and Information Technologies, vol.5, no.2, pp. 1642-1647, (2014)

[12] R. Madhuri, P. Pushpa Latha and K. Prasad Rao, "Weighted Association Rule Mining without Predetermined Weights", International Journal of Engineering Research \& Technology, vol.2, no.2 February (2013)

[13] K. T. Bhandwalkar and M. Bhonsle," Study of Infrequent itemset Mining Techniques", International Journal of Engineering Research and General Science, vol. 2, no. 6, October-November, (2014), ISSN 2091-2730

[14] S. Kamepalli, R.R. Kurra and Y.K. Sundara Krishna, "Apriori Based: Mining Infrequent and Non-Present Itemsets from Transactional Data Bases”, International Journal of Electrical \& Computer Science (IJECSIJENS), vol.14, no.03

[15] L. Szathmary, P. Valtchev, A. Napoli, and R. Godin, "Efficient Vertical Mining of Minimal Rare Itemsets", pp.269-280, (2012) 
[16] A. Gupta, A. Mittal, and A. Bhattacharya, "Minimally Infrequent Itemset Mining Using Pattern-Growth Paradigm and Residual Trees", Proceedings International Conference on Management of Data (COMAD), pp. 57-68, (2011).

[17] L. Szathmary, A. Napoli and P. Valtchev, "Towards rare itemset mining”, in Proceedings of the 19th IEEE International Conference on Tools with Artificial Intelligence, (2007), vol.1, pp.305-312.

[18] Y. Kim, W. Kim, and U. Kim, "Mining Frequent Itemsets with Normalized Weight in Continuous Data Streams", Journal of Information Processing Systems, vol.6, no.1, March (2010).

[19] L. Cagliero and P. Garza, "Infrequent Weighted Itemset Mining Using Frequent Pattern Growth", IEEE Transactions on Knowledge and Data Engineering, pp. 1- 14, (2013).

[20] S. Nathiarasan, Kalaiyarasi, and Manikandan, "Literature Review on Infrequent Itemset Mining Algorithm", International Journal of Advanced Research in Computer and Communication Engineering, vol. 3, no. 8, August (2014).

[21] J. Pillai and O.P. Vyas, "Overview of Itemset Utility Mining and its Applications", International Journal of Computer Applications, vol. 5, no.11, August (2010).

[22] L. Szathmary, A. Napoli and P. Valtchev, "Towards Rare Itemset Mining", 19th IEEE International Conference on Tools with Artificial Intelligence (ICTAI '07), Oct 2007, Patras, Greece, vol. 1, pp.305-312, (2007).

[23] G.C.Lan, T.P.Hong and V.S. Tseng, “A Novel Algorithm for Mining Rare-Utility Itemsets in a MultiDatabase Environment", The 26th Workshop on Combinatorial Mathematics and Computation Theory, pp. 293-302, (2009).

[24] M. Adda, L. Wu and Y. Feng, "Rare Itemset Mining", Sixth International Conference on Machine Learning and Applications, (2007), pp 73- 80.

[25] S. Tsang and U. Dayal, "Data Warehousing and Knowledge Discovery", 13th International Conference, DaWaK 2011, Toulouse, France, August 29-September 2,(2011).

[26] A. Cuzzocrea and U. Dayal, "RP-Tree: Rare Pattern Tree Mining", 13th International Conference, DaWaK 2011, Toulouse, France, August 29-September 2,(2011). Proceedings, pp 277-288.

[27] J. Pillai1, O.P. Vyas and M. K. Muyeba, Internationa Journal on Recent Trends in Engineering and Technology, vol. 10, no. 1, Jan, (2014). 
A Survey on High Utility Rare Itemset Mining

This page is empty by intention. 